\title{
Acute tuberculous myopericarditis mimicking acute myocardial infarction: A case report and literature review
}

\author{
MANYI REN ${ }^{1}$, CHUNSHENG ZHANG $^{2}$, XIAOJUAN ZHANG ${ }^{2}$ and JINGQUAN ZHONG ${ }^{3}$ \\ ${ }^{1}$ Department of Cardiology, Shandong Provincial Qianfoshan Hospital, Shandong University, \\ Jinan, Shandong 250014; ${ }^{2}$ Department of Cardiology, Shandong Provincial Chest Hospital, \\ Jinan, Shandong 250013; ${ }^{3}$ Key Laboratory of Cardiovascular Remodeling and Function Research, \\ Chinese Ministry of Education and Chinese Ministry of Health, Qilu Hospital of Shandong University, \\ Jinan, Shandong 250012, P.R. China
}

Received December 11, 2014; Accepted February 19, 2016

DOI: 10.3892/etm.2016.3187

\begin{abstract}
A number of cases of acute myopericarditis mimicking acute myocardial infarction (AMI) have previously been reported in the literature. However, to the best of our knowledge, such a case resulting from Mycobacterium tuberculosis infection has not previously been described. The present study reports the case of a 21-year-old male patient presenting with acute chest pain, in whom focal ST-segment elevation and elevated cardiac enzymes mimicked a diagnosis of AMI. However, acute tuberculous myopericarditis was diagnosed on the basis of a variety of imaging examinations, laboratory tests, as well as the changes observed in electrocardiograms (ECGs) and in the cardiac enzyme levels. The case highlights the importance of a detailed collection of medical history, comprehensive explanations of serial ECGs, thoracic computed tomography, echocardiogram and coronary angiography in the diagnosis and differentiation of acute tuberculous myopericarditis mimicking AMI.
\end{abstract}

\section{Introduction}

Mycobacterium tuberculosis (M. tuberculosis) is a small, rod-shaped, aerobic, non-spore-forming bacillus and the causative agent of the majority of cases of tuberculosis (1). Humans are the only known reservoirs of M.tuberculosis (1). The most frequently used diagnostic methods for tuberculosis include the tuberculin skin test, acid-fast stain and chest radiographs (1). M. tuberculosis infections may result in myocarditis (2),

Correspondence to: Professor Jingquan Zhong, Key Laboratory of Cardiovascular Remodeling and Function Research, Chinese Ministry of Education and Chinese Ministry of Health, Qilu Hospital of Shandong University, 107 Wen Hua Xi Lu, Jinan, Shandong 250012, P.R. China

E-mail: zjq_dr@163.com

Key words: myopericarditis, acute myocardial infarction, tuberculosis, electrocardiogram, troponin I pericarditis (3) and myopericarditis (4). An anatomical predilection for the right-side mediastinal lymph nodes has been described in cardiac tuberculosis, such that the right side of the heart is the most vulnerable area of the myocardium owing to the potential for contiguous spread (5).

Myopericarditis is primarily a 'pericarditic syndrome' in which acute pericarditis is often accompanied by a certain degree of myocarditis (6). The incidence of myopericarditis was $14.6 \%$ in a clinical series of 274 consecutive cases of acute pericarditis (7). Its typical symptoms include fever, fatigue, pleuritic chest pain, a decreased exercise capacity, and palpitations secondary to cardiac arrhythmias (6). In addition, patients with acute myopericarditis may present with chest pain, focal ST-segment elevation and significant elevation of cardiac enzyme levels, thus mimicking acute myocardial infarction (AMI) $(6,8)$. Therefore, the differentiation of acute myopericarditis from AMI is of great importance due to differences in the complications, treatment strategies and prognoses between the two conditions. A large number of studies have investigated the differentiation between acute myopericarditis and AMI since 2008 (6,8-18). However, to the best of our knowledge, acute myopericarditis resulting from $M$. tuberculosis infection and simulating AMI has not previously been reported. The present study reports the case of a 21-year-old male patient with acute tuberculous myopericarditis in whom focal ST-segment elevation and elevated cardiac enzymes were detected and mimicked AMI.

\section{Case report}

A 21-year-old male patient presented at the Emergency Department of Shandong Provincial Chest Hospital (Jinan, China) in April 2014 with a sharp and nonradiating substernal chest pain lasting for $30 \mathrm{~min}$. The pain increased with inspiration and upon lying in supine position, while it decreased significantly by leaning forward. No rash, palpitation, dyspnea, nausea, vomiting and diarrhea were reported. The patient smoked 10 cigarettes daily for the last 4 years. In addition, the patient had experienced fever, coughing, expectoration and night sweats 2 weeks prior to admission, and had been treated with antibiotics on the basis of a presumptive diagnosis of 

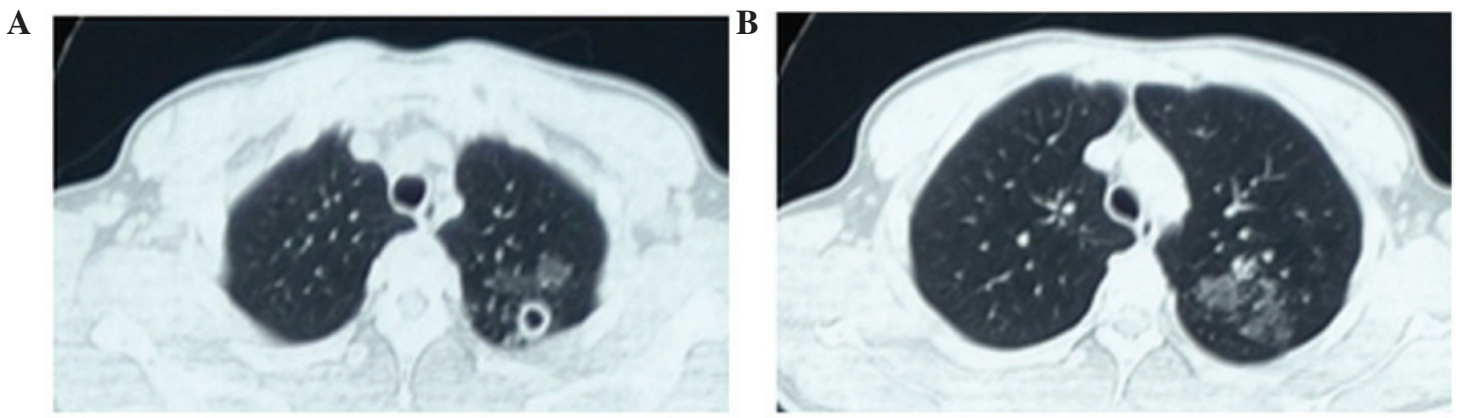

Figure 1. Findings of thoracic computed tomography scanning in April 2014. (A) Small round thick-walled cavities were observed in the apex of the left lung and (B) multiple patchy ground-glass opacities were detected in the left upper lobe of the lung.

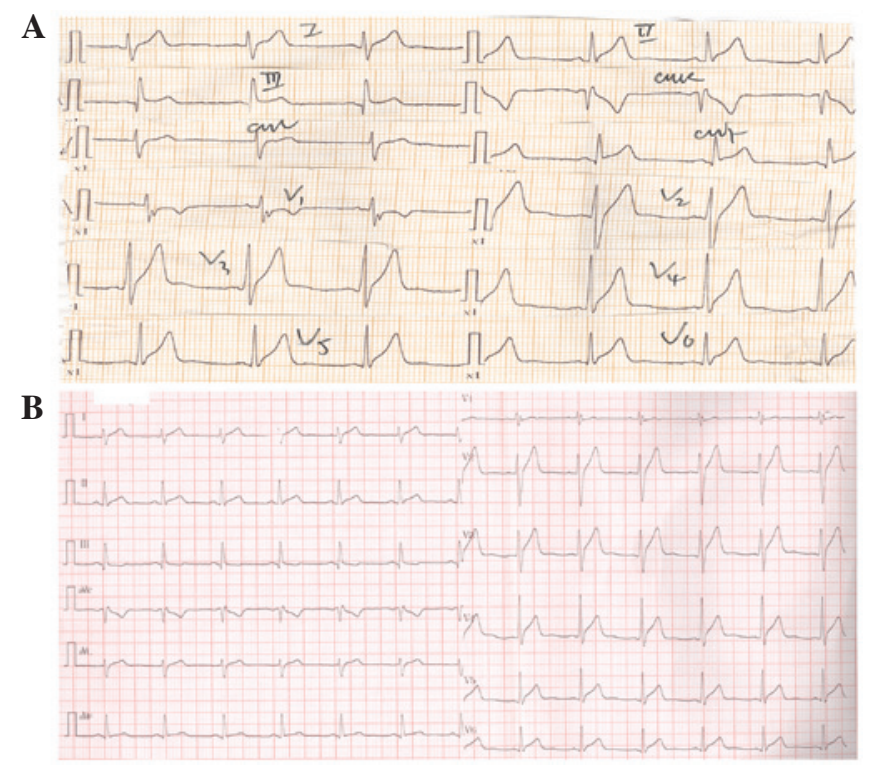

Figure 2. (A) Initial ECG revealed a 1-4-mm ST-segment elevation with coved upward pattern in the inferior leads (II, III and AVF) and lateral precordial leads $\left(\mathrm{V}_{5}\right.$ and $\left.\mathrm{V}_{6}\right)$, as well as a $0.5-\mathrm{mm}$ PR segment depression in lead II. (B) The second ECG without chest pain in the coronary care unit showed tall positive $\mathrm{T}$ waves in leads $\mathrm{V}_{2}-\mathrm{V}_{6}$. $\mathrm{ECG}$, electrocardiogram.

upper respiratory tract infection at a local clinic. However, the symptoms did not subside significantly. No history of hyperlipidemia, hypertension, diabetes and cocaine abuse, or family history of premature coronary artery diseases was reported.

Upon presentation, the patient's vital signs were normal: Body temperature, $37.2^{\circ} \mathrm{C}$; heart rate, 72 beats $/ \mathrm{min}$; respiratory rate, 17 breaths/min; blood pressure, $115 / 70 \mathrm{~mm} \mathrm{Hg}$; and oxygen saturation in room air, 99\%. However, auscultation revealed crackles in the left upper lung fields. The patient's heart rate was regular and heart sounds were normal, with no murmurs, clicks, gallops or rubs. Thoracic computed tomography $(\mathrm{CT})$ was performed using a GE LightSpeed VCT 64 slice CT scanner (GE Healthcare Bio-Sciences, Pittsburgh, PA, USA) and detected small round thick-walled cavities in the apex of the left lung and multiple patchy ground-glass opacities in the left upper lobe of lung (Fig. 1).

A preliminary diagnosis of inferolateral myocardial infarction was presumed on the basis of an abnormal electrocardiogram (ECG; FX-8322; Beijing Fukuda Denshi Medical Instruments Co., Ltd., Beijing, China) performed at the
A

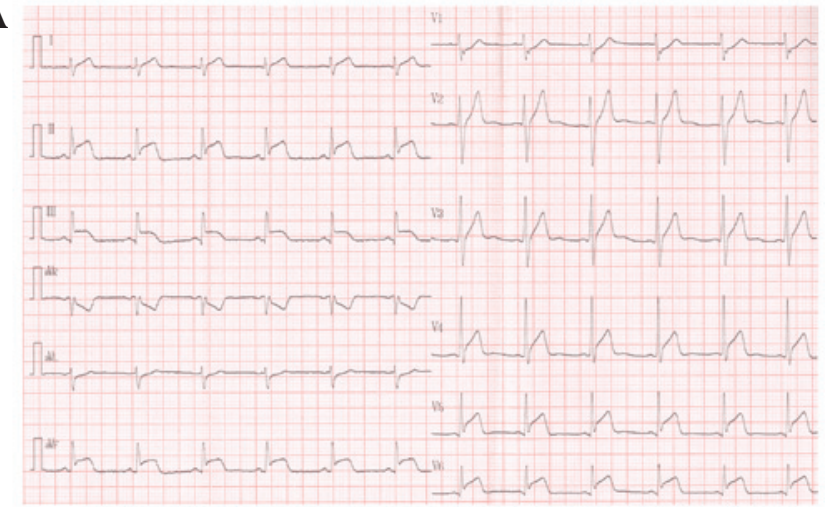

B

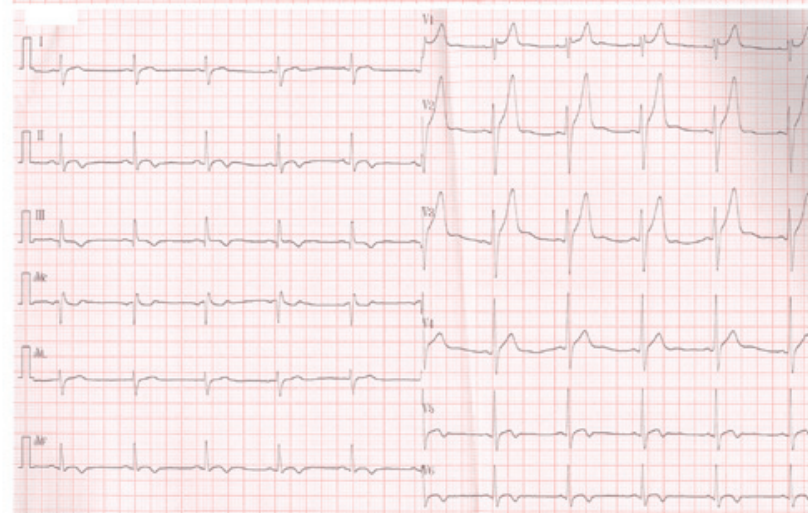

Figure 3. (A) An electrocardiogram revealed 3-6-mm ST-segment elevation with coved upward pattern in leads II, III, AVF, and $\mathrm{V}_{4}-\mathrm{V}_{6}$ at $\sim 14 \mathrm{~h}$ after admission. (B) The elevated ST-segment resolved increasingly and the $\mathrm{T}$ waves begin to invert in the inferolateral leads with tall and prominent positive $\mathrm{T}$ waves in leads $\mathrm{V}_{1}-\mathrm{V}_{3}$ in May 2014.

Emergency Department. Subsequent to sublingual treatment with $0.5 \mathrm{mg}$ nitroglycerin (Shandong Xinyi Pharmaceutical Co., Ltd., Dezhou, China), the patient was transferred to the Coronary Care Unit (CCU) of the Shandong Provincial Chest Hospital with relief of chest pain within $20 \mathrm{~min}$ following admission. The initial ECG revealed a $1-4-\mathrm{mm}$ ST-segment elevation with coved upward pattern in the inferior leads (II, III and AVF) and lateral precordial leads $\left(\mathrm{V}_{5}\right.$ and $\mathrm{V}_{6}$ ), as well as a 0.5 -mm PR segment depression in lead II (Fig. 2A). The second ECG performed in the CCU when the patient was not presenting chest pain showed tall positive $\mathrm{T}$ waves in leads $\mathrm{V}_{2}-\mathrm{V}_{6}$ (Fig. 2B). The patient was found to have a history of exposure to M. tuberculosis upon more detailed investigation. 

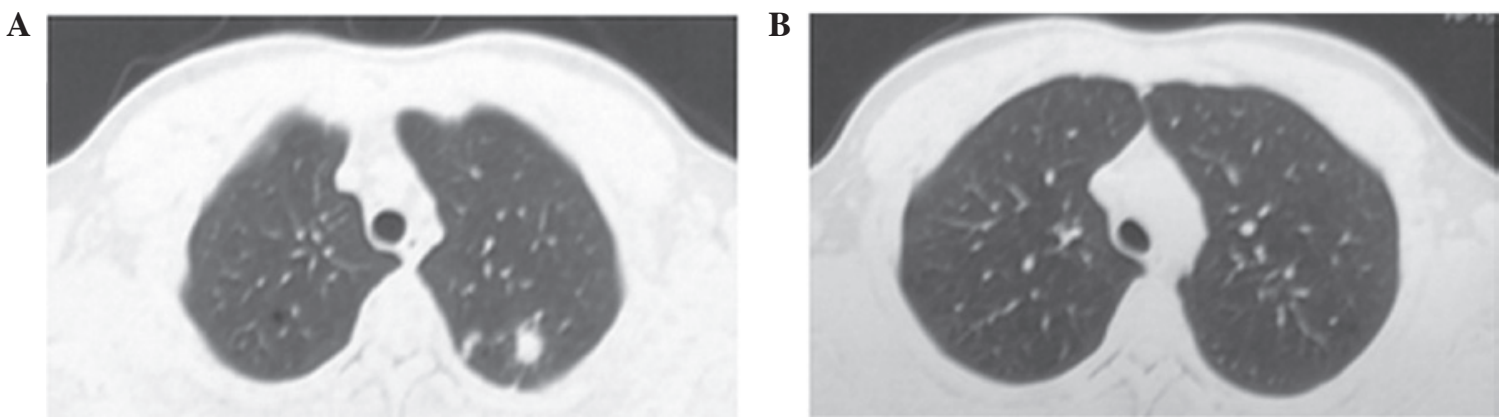

Figure 4. Findings of thoracic computed tomography (CT) scanning in October 2014. (A and B) Patchy shadows of high density were observed in the left upper lobe of the lung, suggesting a significant improvement when compared with the initial CT scans prior to antituberculosis therapy.

Laboratory evaluation revealed that the erythrocyte sedimentation rate (ESR) was $50 \mathrm{~mm} / \mathrm{h}$ (normal range, $0-15 \mathrm{~mm} / \mathrm{h}$ ), the levels of C-reactive protein (CRP) were $37.2 \mathrm{mg} / 1$ (normal range, $0-8.0 \mathrm{mg} / \mathrm{l}$ ), and the cardiac enzyme levels were significantly elevated, with cardiac troponin I (cTnI) at $1.32 \mu \mathrm{g} / \mathrm{l}$ (normal level, $<0.01 \mu \mathrm{g} / \mathrm{l}$ ) and creatine kinase-MB (CKMB) fraction at $26.3 \mathrm{ng} / \mathrm{ml}$ (normal level, <5.1 ng/ml). Other laboratory values were found to be normal. A transthoracic echocardiogram showed slight global hypokinesis with left ventricular ejection fraction of 55\% (normal range, 50-70\%).

All the aforementioned findings favored the diagnosis of acute myopericarditis and pulmonary tuberculosis. Therefore, the patient was administered indomethacin $(50 \mathrm{mg}$, thrice daily for 10 days; Shanxi Yunpeng Pharmaceutical Co., Ltd., Linfen, China), imidapril (2.5 mg, once daily for 1 month; Tianjin Tianbian Pharmaceutical Co., Ltd., Tianjin, China) and metoprolol (6.25 mg, twice daily for 1 month; AstraZeneca, Wuxi, China), along with antituberculosis therapy, including isoniazid (300 mg, once daily), rifampicin (450 mg, once daily), pyrazinamide (750 mg, twice daily) and ethambutol (750 mg, once daily; all Shanghai Sine Pharmaceutical Co., Ltd., Shanghai, China) for 2 months, followed by isoniazid (300 mg, once daily) and rifampicin (450 mg, once daily) for a further 10 months.

Approximately $14 \mathrm{~h}$ after admission, the patient experienced a sudden relapse of squeezing chest pain. A repeat ECG revealed 3-6-mm ST-segment elevation with coved upward pattern in leads II, III, AVF and $\mathrm{V}_{4}-\mathrm{V}_{6}$ (Fig. 3A). The patient received $50 \mathrm{mg}$ indomethacin orally and $3 \mathrm{mg}$ morphine (Shenyang First Pharmaceutical Factory, Shenyang, China) intravenously. The chest pain subsided within $5 \mathrm{~min}$ and did not recur. On the following day, a coronary angiography (CAG; GE Innova 2000; GE Healthcare Bio-sciences) revealed normal coronary anatomy and no ischemia or occlusions. Additionally, sputum acid-fast staining was highly positive.

Serum cTnI and CKMB levels rose to a maximum of $2.95 \mu \mathrm{g} / \mathrm{l}$ and $65.19 \mathrm{ng} / \mathrm{ml}$, respectively, within $26 \mathrm{~h}$ after admission, and returned simultaneously to the normal levels at day 11. The tuberculin skin test (Chengdu Institute of Biological Products Co., Ltd., Chengdu, China) with a 22-mm diameter induration was strongly positive at day 4 , and the patient's temperature fluctuated between $35.8^{\circ} \mathrm{C}$ and $38.4^{\circ} \mathrm{C}$ for 6 days and normalized after day 7 of admission. Sputum culture yielded M.tuberculosis after 20 days of incubation and was sensitive to all antituberculous agents, while no pathogens were isolated in blood cultures.

During the subsequent 3-week hospitalization period, the symptoms of the patient subsided gradually. The elevated ST-segment was gradually resolved, and the $\mathrm{T}$ waves began to invert in the inferolateral leads with tall and prominent positive $\mathrm{T}$ waves in leads $\mathrm{V}_{1}-\mathrm{V}_{3}$ in May 2014 (Fig. 3B). The CRP level and ESR returned to normal, and the echocardiogram was normalized, with an ejection fraction of $66 \%$. A sputum smear was negative for acid-fast bacilli. Furthermore, serum viral titers for Coxsackie virus, adenovirus, Epstein-Barr virus, cytomegalovirus, respiratory syncytial virus, hepatitis $\mathrm{A}, \mathrm{B}$ and $\mathrm{C}$ virus, as well as human immunodeficiency virus (HIV), were all negative. A variety of immunological tests [Euroimmun (Hangzhou) Medical Laboratory Diagnostics Co., Ltd., Hangzhou, China], including assays for rheumatoid factor and anti-nuclear antibodies, such as anti-dsDNA, anti-Sm, anti-ribonucleoprotein, anti-Ro, anti-La, anti-Jo-1 and anti-Scl-70, anti-neutrophil cytoplasmic antibodies and anti-cyclic citrullinated peptide antibody, excluded autoimmune disease.

In May 2014, the patient was discharged approximately 23 days after admission with instructions to take imidapril (2.5 $\mathrm{mg}$, once daily) and metoprolol (6.25 mg, twice daily) for 1 week, as well as antituberculous drugs as mentioned above. In addition, the patient was advised to limit his physical activity for 4 weeks. During the 7-month follow-up period, the patient remained asymptomatic and the ECG was normal. Thoracic CT scans revealed patchy shadows of high density in the left upper lobe of lung (Fig. 4), suggesting a significant improvement over the initial CT prior to antituberculosis therapy. Following the withdrawal of isoniazid and rifampicin in June 2015, the patient recovered well and had normal CT lung images. Written informed consent was obtained from the patient for the publication of this study.

\section{Discussion}

In clinical practice, pericarditis and myocarditis coexist since they share common etiologic agents. However, these two conditions are rarely of equivalent intensity, resulting in clinical syndromes that are mainly 'pericarditic' or 'myocarditic'. Myopericarditis indicates a primarily pericarditic syndrome with minor degrees of myocardial involvement, whereas the term perimyocarditis suggests a mainly myocarditic syndrome (6). 


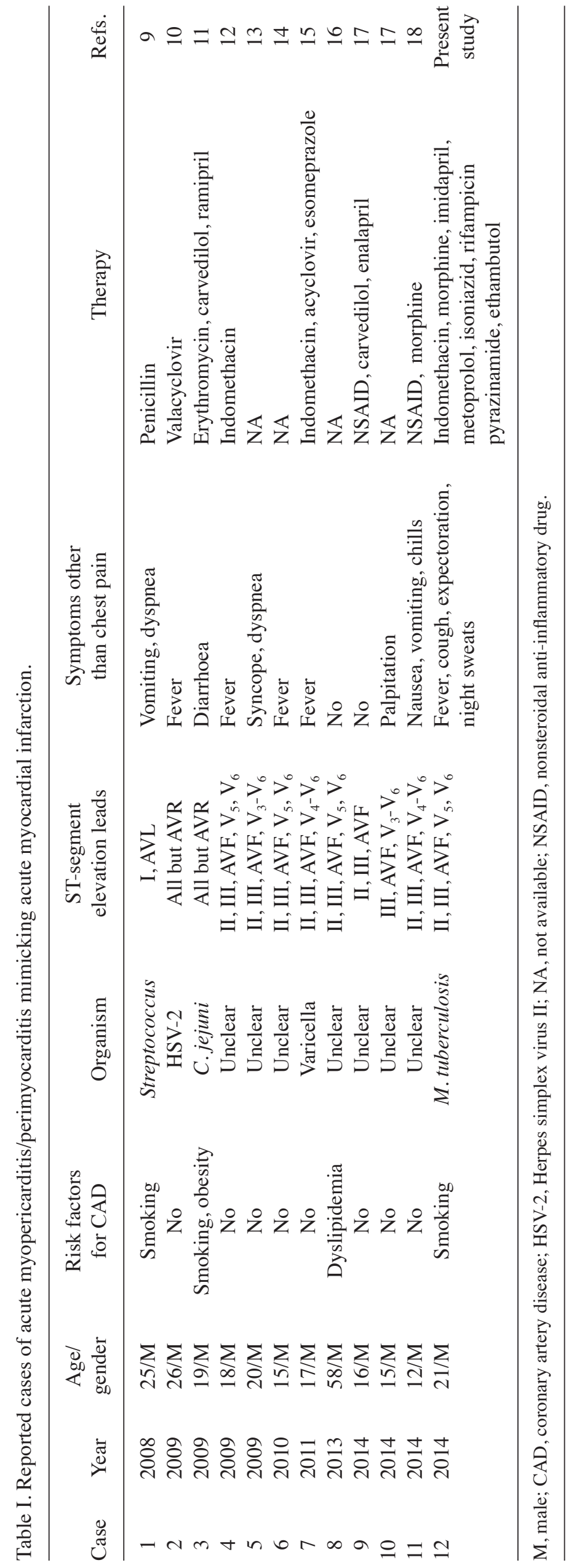


The most common cause of myopericarditis is viral infection, followed by bacterial infection. Among bacterial causes, tuberculosis is possibly the most important cause worldwide due to the high number of cases in developing countries, particularly in the setting of HIV coinfection $(19,20)$. Tuberculous pericarditis caused by M. tuberculosis is found in $\sim 1 \%$ of all autopsied cases of tuberculosis and in $1-2 \%$ of cases with pulmonary tuberculosis (21). In the present case, tubercle bacilli detected in a sputum smear and culture demonstrated indirectly the diagnosis of tuberculous myopericarditis based on the exclusion of viral infection, other bacterial infections and autoimmune disease. Although a previous case of tuberculous myopericarditis has recently been published (4), to the best of our knowledge, the present study reports the first case of acute tuberculous myopericarditis mimicking AMI.

The present study performed a literature search to identify previous reports of acute myopericarditis or perimyocarditis mimicking AMI. The following search terms in 'Titles and Abstracts' were used to retrieve relevant articles from Scopus (http://www.scopus.com), MEDLINE (http://www.ncbi.nlm. nih.gov/pubmed) and ScienceDirect (www.sciencedirect.com) databases from inception to December 2014: 'myopericarditis or perimyocarditis' and 'mimicking or simulating or masquerading' and 'myocardial infarction or acute coronary syndrome' and 'English'. The wildcard term '*' was used to increase the sensitivity of the search strategy. Only the literature denoting myopericarditis or perimyocarditis mimicking AMI were included. A total of 11 cases (9-18) of acute myopericarditis or perimyocarditis mimicking AMI (Table I) were identified by this search, of which there was 1 case with Streptococcus infection, 1 case with Campylobacter jejuni, 1 case with herpes simplex virus II and 1 case with varicella, whereas unclear causes were reported in the remaining cases. All patients in previous cases were males aged 12-26 years old, with the exception of a 58-year-old man. One or more risk factors for coronary artery disease (CAD), included smoking, obesity and dyslipidemia, were found in 3 patients.

Myopericarditis can be clinically defined as a definite diagnosis of acute pericarditis and elevation of cardiac markers of injury (cTnI or troponin T, and CKMB fraction), without further focal or diffuse depressed left ventricular function observed on a echocardiogram or cardiac magnetic resonance (CMR) scan (8). In the present study, the patient's symptoms of sharp, positional chest pain and minimal pericardial effusion were suggestive of acute pericarditis. The levels of cTnI and CKMB were markedly elevated, while the left ventricular function was normal. These observations are in accordance with the clinical diagnosis of myopericarditis. However, the focal ST segment elevation in inferolateral leads resulted in difficulties in distinguishing acute myopericarditis from AMI. A typical pattern of ECG evolution includes diffuse ST elevation and PR depression, followed by normalization of the ST and PR segments, and subsequent diffuse T-wave inversions (22). Imazio et al (7) demonstrated that certain findings occurred significantly more frequently in patients with myopericarditis compared with those in acute pericarditis patients, and these findings included: Atypical ECG changes and T-wave inversion prior to ST-segment normalization; and cardiac arrhythmias, including supraventricular or ventricular ectopic beats, as well as nonsustained ventricular tachycardia.
Although a triad of acute chest pain, elevated cardiac enzymes and localized ST-segment elevation in inferolateral leads mimicked AMI, the present patient had a concave upwards morphology of the ST-segment in leads II, III, AVF and $\mathrm{V}_{5}-\mathrm{V}_{6}$ and a PR-segment deflection opposite to P-wave polarity in lead II, suggesting a diagnosis of myopericarditis. ST-segment elevations were almost localized in inferolateral leads of the 11 previously-reported cases (Table I). Electrocardiographic abnormalities associated with acute myopericarditis have been previously attributed to changes in repolarization involving the ventricle and atrium, which are due to epicardial inflammation. These repolarization changes consequently affect the morphology of the PR segment, ST segment, and T wave (22).

Differential diagnosis of acute myopericarditis based on the ECG findings included benign early repolarization (BER) and AMI (22). A previous study found that the morphology of ST segment elevation in BER patients is similar to that of acute myopericarditis, presenting an indistinct $\mathrm{J}$ point and an initial concave upsloping. Unlike in acute myopericarditis, the ST segment elevation in BER is mainly observed in the precordial leads, with most prominent appearance in the right precordial leads and less commonly in lead $\mathrm{V}_{6}$ (where the ST segment is frequently isoelectric) (23). Furthermore, ST segment elevation is often short term in acute myopericarditis, whereas its short-term resolution is not observed in BER and it may transiently return to the baseline upon exercise. However, differentiating between AMI and acute myopericarditis on an ECG can be challenging. Regarding the ST morphology, the elevation is initially convex in AMI, whereas it is concave in acute myopericarditis. In addition, Q waves may be observed in AMI, however, they are rarely reported in acute myopericarditis ECGs. By contrast, PR segment depression suggests a diagnosis of acute myopericarditis rather than AMI, as indicated by positional chest pain (22).

In the present case, a number of evidence favored the diagnosis of myopericarditis, including the young age of the patient, the single risk factor for CAD (smoking 10 cigarettes daily for 4 years), history of pulmonary tuberculous infection, concave upward ST segment elevation, PR segment depression and the absence of regional wall motion abnormalities on an echocardiogram. However, the elevated cardiac enzyme levels and the ST segment elevation in inferolateral leads mimicked a diagnosis of AMI, which was subsequently excluded by CAG examination and echocardiography.

Other imaging examinations for the differentiation between acute myopericarditis and AMI include contrast-enhanced CMR, 64-slice CT coronary angiography and endomyocardial biopsy. In acute myocarditis, CMR shows an abnormal patchy myocardial signal with delayed enhancement suggesting myonecrosis and edema, without presenting in the subendocardial region, which exclude the diagnosis of CAD (24). In acute myopericarditis, similar findings upon the use of CMR further confirm that the subepicardial myocardium is involved and the subendocardial region is spared $(13,16,17)$. Although biopsy is the main technique used for the diagnosis of myocarditis, it may be of limited clinical value in certain cases, particularly in myopericarditis, where pericardial involvement is prevalent. In the 11 identified cases of acute myopericarditis mimicking AMI (9-18), endomyocardial biopsy of the right ventricle 
was only performed in 1 case, and the results demonstrated a number of the expected histological features of myocarditis, including endomyocardial fibrosis, myocytolytic changes and interstitial edema (14).

The management of acute myopericarditis is primarily aimed at the pathogen, pericarditis and myocarditis, with the exception of the recommendation of rest and avoiding of physical activity for 4-6 weeks (6). Therapy for the pathogen involves the use of antiviral drugs, antibiotics or antituberculosis medications (6). In the absence of significant myocardial failure, the management of myopericarditis is similar with that in acute pericarditis, with nonsteroidal anti-inflammatory drugs (NSAIDs) being the commonly administered therapy (6) Ibuprofen may be the preferred NSAID since side effects are rare, and it has a favorable impact on coronary artery blood flow and a large dose range (25). Other medications lacking evidence-based data in myopericarditis include corticosteroid, colchicine and intravenous immunoglobulin (6). In acute myopericarditis with decreased left ventricular ejection fraction, treatment includes myocardial protection and inhibition of cardiac remodeling. In the present case, the prescribed therapeutic measures, including rest, indomethacin, imidapril, metoprolol and antituberculosis agents, were completely performed and the patient recovered well.

In conclusion, the current study reported for the first time a case of acute tuberculous myopericarditis mimicking AMI in a 21-year-old male patient. The case highlights the importance of a detailed collection of medical history, comprehensive explanations of serial ECGs, thoracic CT scan, echocardiogram and CAG in the diagnosis and differentiation of acute tuberculous myopericarditis mimicking AMI.

\section{Acknowledgements}

The present study was sponsored by grants from the Natural Science Foundation of China (no. 81270238) and the Scientific Research Foundation for the Doctoral Degree, State Education Ministry of China (no. 20100131110059), and was supported by the Scientific Development Plan of Shandong Province of China (no. 2012G0021850).

\section{References}

1. Goldman L and Schafer AI (eds): Tuberculosis. In: Goldman-Cecil Medicine. Vol 1. 25th edition. Elsevier Saunders, Philadelphia, PA, pp2030-2037, 2014.

2. Michira BN, Alkizim FO and Matheka DM: Patterns and clinical manifestations of tuberculous myocarditis: A systematic review of cases. Pan Afr Med J 21: 118, 2015.

3. Lazaros G and Tousoulis D: Tuberculous Pericarditis: A Complex Puzzle to Put Together. EBioMedicine 2: 1570-1571, 2015.

4. Desai N, Desai S, Chaddha U and Gable B: Tuberculous myopericarditis: A rare presentation in an immunocompetent host. BMJ Case Rep 2013, 2013.
5. Maeder M, Ammann P, Rickli H and Schoch OD: Fever and night sweats in a 22-year-old man with a mediastinal mass involving the heart. Chest 124: 2006-2009, 2003.

6. Imazio $M$ and Trinchero R: Myopericarditis: Etiology, management and prognosis. Int J Cardiol 127: 17-26, 2008.

7. Imazio M, Cecchi E, Demichelis B, Chinaglia A, Ierna S, Demarie D, Ghisio A, Pomari F, Belli R and Trinchero R: Myopericarditis versus viral or idiopathic acute pericarditis. Heart 94: 498-501, 2008

8. Imazio M and Cooper LT: Management of myopericarditis. Expert Rev Cardiovasc Ther 11: 193-201, 2013.

9. Khavandi A, Whitaker J, Elkington A, Probert J and Walker PR: Acute streptococcal myopericarditis mimicking myocardial infarction. Am J Emerg Med 26: 638.e1-e2, 2008.

10. Tian W, Zhang Z, Bai X, Zeng D and Qi G: Acute myopericarditis masquerading as acute myocardial infarction. J Nanjing Med Uni 22: 130-133, 2008.

11. Lai T, Yadav R and Schrale R: Mimicking myocardial infarction: Localized ST-segment elevation in Campylobacter jejuni myopericarditis. Intern Med J 39: 422-423, 2009.

12. Omar HR, Fathy A, Rashad R and Elghonemy M: Acute perimyocarditis mimicking transmural myocardial infarction. Int Arch Med 2: 37, 2009.

13. Poh KK, Chan EH, Chia BL and Chai P: Acute perimyocarditis masquerading as acute coronary syndrome with spontaneous resolution of increased left ventricular wall thickness. Ann Acad Med Singapore 38: 278-279, 2009.

14. Nisbet BC and Breyer M: Acute myopericarditis with focal ECG findings mimicking acute myocardial infarction. J Emerg Med 39: e153-e158, 2010.

15. De A, Myridakis D, Kerrigan M and Kiblawi F: Varicella myopericarditis mimicking myocardial infarction in a 17-year-old boy. Tex Heart Inst J 38: 288-290, 2011.

16. Bolognesi $\mathrm{M}$ and Bolognesi D: Acute coronary syndrome vs. myopericarditis-not always a straightforward diagnosis. Am J Case Rep 14: 221-225, 2013.

17. Gupta SK and Naheed Z: Chest pain in two athletic male adolescents mimicking myocardial infarction. Pediatr Emerg Care 30: 493-495, 2014.

18. Sharma J, Fernandes N, Alvarez D and Khanna S: Acute myopericarditis in an adolescent mimicking acute myocardial infarction. Pediatr Emerg Care 31: 427-430, 2015.

19. Mayosi BM: Contemporary trends in the epidemiology and management of cardiomyopathy and pericarditis in sub-Saharan Africa. Heart 93: 1176-1183, 2007.

20. Krauss MR, Harris DR, Abreu T, Ferreira FG, Ruz NP, Worrell C and Hazra R; NISDI Pediatric Study Group: Tuberculosis in HIV-infected infants, children and adolescents in Latin America. Braz J Infect Dis 19: 23-29, 2015.

21. Fowler NO: Tuberculous pericarditis. JAMA 266: 99-103, 1991.

22. Chan TC, Brady WJ and Pollack M: Electrocardiographic manifestations: Acute myopericarditis. J Emerg Med 17: 865-872, 1999.

23. Shabetai R: Acute pericarditis. Cardiol Clin 8: 639-644, 1990.

24. Kern J, Modi R, Atalay MK and Kochilas LK: Clinical myocarditis masquerading as acute coronary syndrome. J Pediatr 154: 612-615, 2009.

25. Maisch B, Seferović PM, Ristić AD, Erbel R, Rienmüller R, Adler Y, Tomkowski WZ, Thiene G and Yacoub MH; Task Force on the Diagnosis and Management of Pricardial Diseases of the European Society of Cardiology: Guidelines on the diagnosis and management of pericardial diseases executive summary; The Task force on the diagnosis and management of pericardial diseases of the European Society of Cardiology. Eur Heart J 25: 587-610, 2004. 